This material is published in the open archive of Mid Sweden University

DIVA http://miun.diva-portal.org to ensure timely dissemination of scholarly and technical work. Copyright and all rights therein are retained by authors or by other copyright holders. All persons copying this information are expected to adhere to the terms and constraints invoked by each author's copyright. In most cases, these works may not be reposted without the explicit permission of the copyright holder.

Yun Li; Sjöström, M.; Olsson, R., "Coding of plenoptic images by using a sparse set and disparities," in 2015 IEEE International Conference on Multimedia and Expo (ICME), June 29 2015-July 32015

http://dx.doi.org/10.1109/ICME.2015.7177510

(C) 2015 IEEE. Personal use of this material is permitted. Permission from IEEE must be obtained for all other uses, in any current or future media, including reprinting/republishing this material for advertising or promotional purposes, creating new collective works, for resale or redistribution to servers or lists, or reuse of any copyrighted component of this work in other works." 


\title{
CODING OF PLENOPTIC IMAGES BY USING A SPARSE SET AND DISPARITIES
}

\author{
Yun Li, Mårten Sjöström, and Roger Olsson \\ Dept. of Information and Communication Systems, Mid Sweden University, Sweden \\ Email: \{yun.li, marten.sjostrom, and roger.olsson\}@miun.se
}

\begin{abstract}
A focused plenoptic camera not only captures the spatial information of a scene but also the angular information. The capturing results in a plenoptic image consisting of multiple microlens images and with a large resolution. In addition, the microlens images are similar to their neighbors. Therefore, an efficient compression method that utilizes this pattern of similarity can reduce coding bit rate and further facilitate the usage of the images. In this paper, we propose an approach for coding of focused plenoptic images by using a sparse image set and disparities, i.e., a sparse plenoptic image set plus disparity maps. Based on this format, a reconstruction method by using interpolation and inpainting is devised to reconstruct the original plenoptic image. As a consequence, instead of coding the original image directly, we encode the sparse image set plus the disparity maps and use the reconstructed image as a prediction reference to encode the original image. The results show that the proposed scheme performs better than HEVC intra with more than $5 \mathrm{~dB}$ PSNR or over 60 percent bit rate reduction.
\end{abstract} sion,

Index Terms - Plenoptic, lightfield, HEVC, compres-

\section{INTRODUCTION}

Plenoptic cameras capture a sampling of the light field with the directions and the intensity of outgoing radiances from a scene. This capturing process enables the capability of image refocusing and multi-view imaging during post-production. However, a densely sampled plenoptic image contains repetitive patterns and has a large resolution. The image can possibly be represented by a subset of its microlens images plus disparities. The question is if plenoptic image can be encoded efficiently by using such a representation.

The concept of plenoptic capturing was introduced by Gabriel Lippmann in 1908 [1]. A plenoptic function, which is also referred to a light field, can be described by using a twoplane representation [2]. Therefore, a light field has four dimensions $(x, y)$, and $(r, t)$, which locate the coordinates of a

This work has been supported by grant 20120328 of the Knowledge Foundation, Sweden, by grant 00174636 of the EU European Regional Development Fund, Mellersta Norrland, Sweden. We also want to acknowledge Todor Georgiev for providing the light field images online. radiance passing through the two planes, respectively. There are several techniques for capturing a light field image, for example, by using multi-camera arrays, coded apertures, and microlens arrays. In the capturing with microlens arrays, two capturing techniques are further derived, which are plenoptic 1.0 and plenoptic 2.0. Cameras with plenoptic 2.0 technique [3] are also referred to as focused plenoptic cameras. By putting the focal plane of microlenses away from the image sensor plane, the focus plenoptic camera captures both angular and spatial information in each microlens image and across microlens images. It provides a trade-off between spatial and angular information for the capturing. We only consider images captured by focused plenoptic cameras in the context of this paper. A densely sampled plenoptic image implies that adjacent microlens images, also referred to as Elemental Images (EI), are highly correlated.

The plenoptic image consists of a grid of EIs whose contents are similar to their neighbors. Therefore, one problem with respect to coding is that the image exhibits repetitive patterns, and a large amount of redundancy exists. Another problem lies in that not all EIs are always needed for rendering. Therefore, we are motivated by the two problems to devise an approach that can: 1) remove the redundancy before coding, 2) encode plenoptic contents efficiently, and 3) provide coding scalability.

There are other techniques to capture a light field image as mentioned. Coding approaches with respect to light field images in general can be applied to plenoptic images. Previous coding work on light field image compression is mainly classified into three categories: vector quantization, predictive coding and progressive coding [4]. For the vector quantization [5] approach, light field images are partitioned into small blocks, which are represented as vectors. A small subset of the vectors is trained to approximate the entire vector space. In predictive coding, an early work [6] arranges light field images into a grid. Images within the grid are recursively predicted from a few intra coded images. The prediction efficiency is further improved by using homography [7]. As to the progressive coding, Discrete Wavelet Transform (DWT) is usually applied to achieve a finer granularity of scalability [4] [8]. Shape Adaptive Discrete Wavelet Transform (SA-DWT) [8] was employed in a wavelet scheme with disparity-compensated lifting and shape adaptation to 
preserve the boundaries of objects in images. As light field images can be considered as 4-D contents, 4-D wavelets were used in [9] for the compression. In addition, there are approaches that do not distinctively lie in any of the categories mentioned above. Examples are compression with distributed coding [10], with Principle Component Analysis (PCA) [11], and with intra displacement prediction [12]. If camera geometries are known, multi-view encoders in general with hierarchical coding structures can be used for coding of plenoptic images. However, an obvious drawback of using multi-view encoders directly is that each EI must be padded to the size of a power of two [13] for feeding into the encoder. Since an EI is very small, the padding will result in an uneglectable amount of extra data to encode. In addition, the coding performance is dependent on the coding structure. The work in [12] has proposed a displacement prediction scheme that efficiently exploits the inter-EIs correlation. But the displacement intra does not provide any scalability for the rendering.

In this paper, we introduce a coding approach for plenoptic images by using a sparse set of EIs and disparities. Approximated camera geometries are assumed to be known, and EIs can be separated from the plenopitc image. We firstly estimate disparities for EIs, and then uniformly retain a sparse set of EIs. Based on the sparse set and disparities, a full plenoptic image is reconstructed by using prediction with interpolation and, for those non-predictable areas, with inpainting. The reconstructed plenoptic image is utilized to predict the original full image by using a modified HEVC encode. The novelties of this paper are: 1) we encode plenoptic images by using a sparse set of EIs and their associated disparities; 2) inpainting and interpolation techniques are applied for the reconstruction of plenoptic images; 3) a modified HEVC encoder is devised for the prediction and coding of plenoptic images from their reconstructed images; 4) we further integrated the proposed scheme into the displacement intra prediction in HEVC; 5) scalability of the proposed coding scheme is also described.

The overall aim of the work is to improve the coding efficiency for plenoptic contents. The work is limited to the coding for densely sampled focused plenoptic images. The goal is to investigate the rate-distortion ratio objectively for the proposed scheme.

The sequence of the paper is organized as follows. We illustrate the displacement intra prediction in Section 2 and the proposed scheme in section 3. Test arrangements and evaluation criteria are presented in Section 4, and Section 5 shows the results and analysis. Section 6 concludes this paper.

\section{DISPLACEMENT INTRA PREDICTION}

For the paper to be self-contained and for a better clarity of presenting our proposed scheme, the displacement intra prediction is briefly described here. The displacement intra prediction scheme in HEVC has been proposed in [12] for the coding of plenoptic images. The scheme can perform a bi- directional prediction in the spatial domain of an image and is referred to as B-coder.

As shown in Fig. 1(a), two parts of the image are assumed as two reference pictures available in the picture reference list $L_{0}$ and $L_{1}$. A current coding block is predicted from the best matching reference block, which can be the best matching block in list $L_{0}$, the best in list $L_{1}$, or $\frac{\left(P_{0}+P_{1}\right)}{2} . P_{0}$ and $P_{1}$ are blocks obtained from $L_{0}$ and $L_{1}$, respectively. The best is measured in terms of minimum rate-distortion. In addition, the original HEVC directional intra prediction is also evaluated in the Rate-Distortion Optimization (RDO) process of HEVC. As a result, the best prediction mode is selected for coding the current block. A detail description of the original

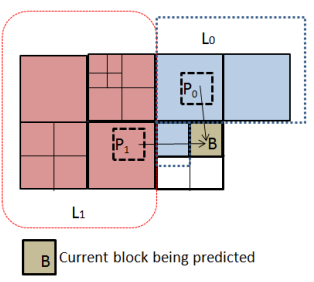

(a) (b)

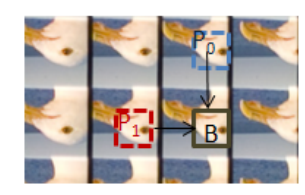

Fig. 1. Bi-prediction within an image. (a) Two parts in color blue and red are assumed as two reference pictures and available in the reference list $L_{0}$ and $L_{1}$; (b) an illustration of the prediction on a light field image.

HEVC intra and the displacement intra can be referred to [13] and [12], respectively.

\section{PROPOSED METHOD}

Fig. 2 and 3 present the overview diagrams of the proposed coding scheme, the details of each block in the diagram are explained in the following subsections.

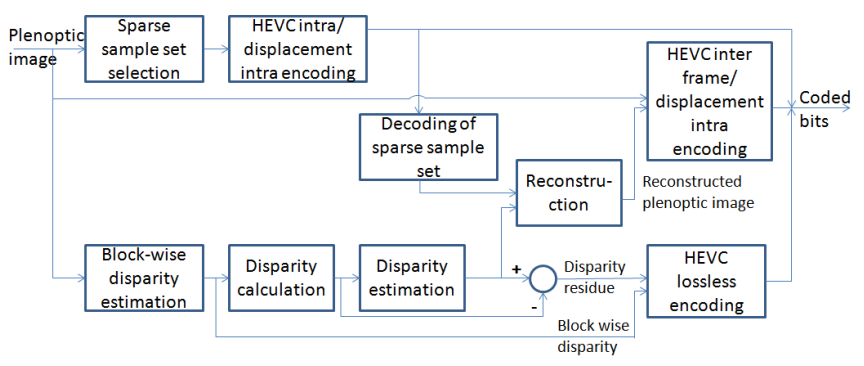

Fig. 2. The proposed plenoptic image encoding system.

\subsection{Encoding}

Sparse sample set selection: A plenoptic image is sampled into a sparse plenoptic image set as illustrated in Fig. 4. Assume $(x, y)$ is the coordinate of an EI $I_{E(x, y)}(r, t)$ within the plenoptic image $C(x, y, r, t)$ in Fig. $4, x \in[1, N]$, 


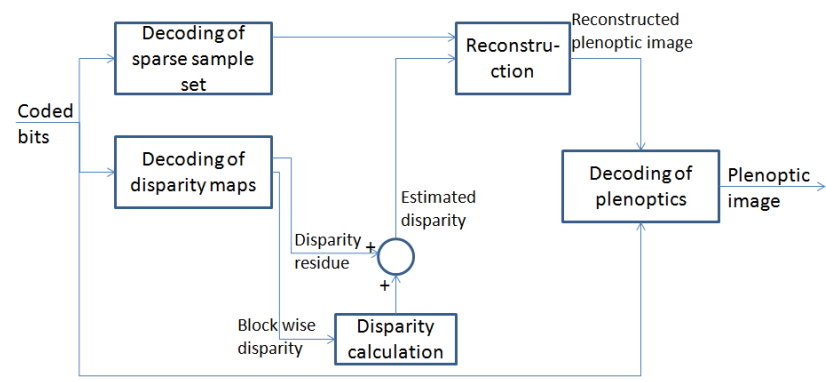

Fig. 3. The proposed plenoptic image decoding system.

$y \in[1, M], r \in\left[1, N_{t}\right]$, and $t \in\left[1, M_{t}\right]$, where $N, M$, $N_{t}$, and $M_{t}$ are the size of each dimension. A sparsely sampled image $C\left(x_{s}, y_{s}, r, t\right)$ is obtained with a sampling factor $s$ such that $\left\{\left(x_{s}, y_{s}\right) \mid x_{s} \in x \cap y_{s} \in y \cap x_{s} \bmod s=\right.$ $\left.\left.0 \cap y_{s} \bmod s=0\right)\right\}$. The sampling process on a captured plenoptic image is illustrated in Fig. 5.

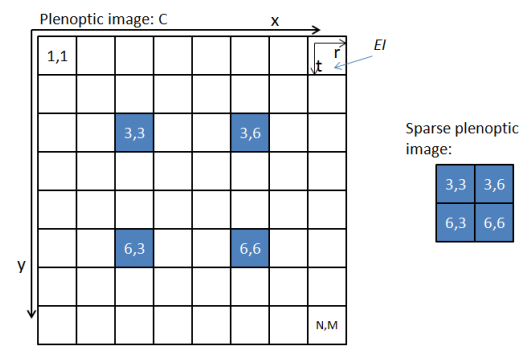

Fig. 4. A 8 by 8 plenoptic image sparsely sampled by a factor of 3 .

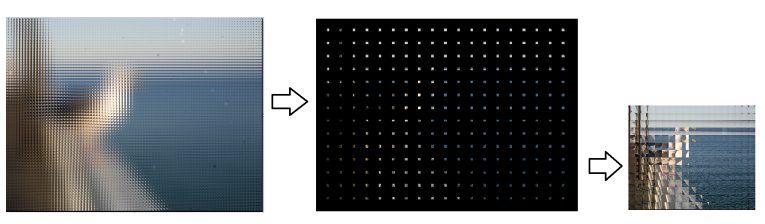

Fig. 5. An example of the sampling.

HEVC intra/displacement intra encoding: The sparsesampled image can be encoded by state of the art image encoders. In this work, we employ two coders, HEVC [13] original intra and the displacement intra B-coder [12] mentioned in the previous section, for the encoding.

Decoding of sparse sample set: The coded image is decoded. These decoded sparse sample set of images are used for a later reconstruction.

Block-wise disparity estimation: As an entire EI is considered as a block, the disparity between the current EI and the EI at its right side is estimated as the horizontal disparity, and the current EI and the EI at its bottom side as the vertical disparity. The estimation is performed by minimizing the
Mean Square Error (MSE) between the two neighboring EIs. The results from the estimation are two disparity maps for the horizontal and the vertical direction. Therefore, two disparity maps with a resolution of only 7 by 7 are produced for the plenoptic image illustrated in Fig. 4.

Disparity calculation: For a later reconstruction, the disparities between all EIs outside the sparse set to each EI in the sparse set with a range of $r$ must be estimated. An EI in the sparse set is located at each coordinate $\left(x_{s}, y_{s}\right)$ within the plenoptic image illustrated in Fig. 6. We refer these disparities to as sparse-set-centered disparities.

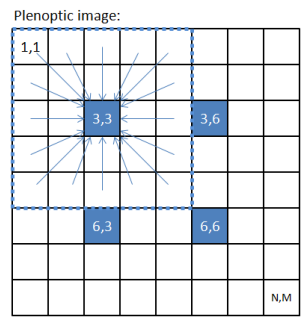

Fig. 6. Disparity calculation from all EIs outside the sparse set in the blue box to an EI centered at $(3,3)$ in the sparse set with a range of $r=2$.

Because the block-wise disparities have already been acquired, the sparse-set-centered disparities can simply be calculated by an addition horizontally and vertically from the block-wise disparities. Assume $D_{h}((x, y),(x+1, y))$ is the block wise horizontal disparity for the EI at the coordinate (x, y) to its neighbor at the right side, and $D_{v}((x, y),(x, y+1))$ is the block wise vertical disparity to its neighbor at the bottom side. The horizontal and the vertical sparse-set-centered disparity for the EI at $(x, y)$ to the EI at $\left(x_{s}, y_{s}\right)$ are calculated by: $D_{h}\left((x, y),\left(x_{s}, y_{s}\right)\right)=$

$$
\begin{aligned}
& \left\{\begin{array}{l}
\sum_{i=x}^{x_{s}-1} D_{h}((i, y),(i+1, y)), x_{s}>x \\
\sum_{i=x_{s}}^{x-1}-D_{h}((i, y),(i+1, y)), x>x_{s},
\end{array}\right. \\
& D_{v}\left((x, y),\left(x_{s}, y_{s}\right)\right)= \\
& \left\{\begin{array}{c}
\sum_{i=y}^{y_{s}-1} D_{v}((x, i),(x, i+1)), y_{s}>y \\
\sum_{i=y_{s}}^{y-1}-D_{v}((x, i),(x, i+1)), y>y_{s} .
\end{array}\right.
\end{aligned}
$$

Disparity estimation: However, the calculated disparities are imprecise. In order to obtain more accurate disparities, a refinement search around the calculated sparse-set-centered disparities is carried out. The search is aiming at minimizing the MSE further between two EIs under consideration, i.e., an EI outside the sparse set to an EI within the sparse set with a range $r$. The arrows in the blue box in Fig. 6 indicate that the refinement is performed for all EIs at coordinate $(x, y)$ to the EI centered at $(3,3)$ in the sparse set within a range of $r=2$. This process is repeated for the EIs centered at $(3,6),(6,3)$, and $(6,6)$. The results are two estimated disparity maps of resolution 10 by 10 for the horizontal and the vertical direc- 
tions.

The rationale for estimating the disparities in two steps, i.e., from the block-wise disparity to the sparse-set-centered disparity, is to increase the accuracy of the disparity estimation. This is because it is likely to find a good match for an EI from its direct neighbors. By first performing an estimation between neighbors and then a refinement, direct disparity estimation between two distant EIs is avoided.

HEVC lossless encoding: Four disparity maps are encoded losslessly by using HEVC intra. The maps are the two block-wise disparity maps and the two disparity residue maps, which result from the subtraction between the estimated and the calculated sparse-set-centered disparity maps.

The four disparity maps have a smooth property, which is also the reason for choosing them as the disparity representation formats for coding. The four maps are encoded independently, because the HEVC intra can reduce the spatial redundancy efficiently. Adding inter frame prediction did not improve the coding performance in our experiment.

Reconstruction: A full plenoptic image is reconstructed from the decoded sparse plenoptic image set. As the Fig. 7 shows, the EIs from the decoded sparse image set are placed into their original coordinates within the full plenoptic image. Based on the estimated sparse-set-centered disparities, the unknown EIs are obtained from the known EIs by a disparity shift. If multiple known EIs are available for an unknown EI within the range $r$, they are averaged. As an example in Fig. $7, \mathrm{EI}$ at coordinate $(1,1)$ is extrapolated from $\mathrm{EI}$ at $(3,3)$, and EI at $(5,4)$ is interpolated from four known EIs.

After the interpolation or extrapolation process, there are still missing areas in each of the reconstructed EIs. Inpainting approaches in general can be used to fill the missing areas. In our work, a fluid dynamic inpainting approach [14] is employed to inpaint the missing areas. This inpainting method assumes that the isophotes in the image as flows. The missing data is filled by solving the Navier-stokes equation.

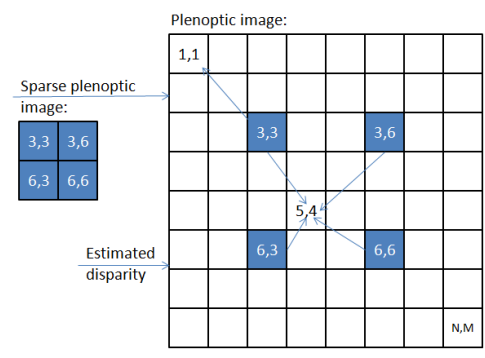

Fig. 7. Reconstruction of plenoptic images with $r=2$. EI at $(1,1)$ is extrapolated, and EI at $(5,4)$ is interpolated.

HEVC inter frame/displacement intra encoding: The HEVC encoder is modified for the prediction of plenoptic images in coding. During the initialization of the modified encoder, the reconstructed plenoptic image from the Reconstruction process is loaded into the picture reference list in
HEVC and available for inter frame prediction. During encoding, both intra prediction and inter prediction are performed, the best coding mode with the RDO for each coding block is chosen for the prediction. Prediction residues are quantized, transformed and entropy encoded as in HEVC. The coded bit-stream is formed together with the lossless coded side information such as prediction vectors and mode signaling flags. The intra prediction used here can also be either HEVC original intra or the displacement intra.

The final coded bit streams consist of three components, which are: 1) coding of sparse sample set from HEVC intra/displacement intra encoding ,2) coding of disparity maps from HEVC lossless coding, and 3) coding of full plenoptics from HEVC inter frame/displacement intra encoding. We name our proposed scheme as 1) proposed scheme and 2) proposed+displacement intra (proposed +Disp.) based on the coding methods for each component listed in Table 1.

Table 1. The coding methods for each component

\begin{tabular}{|l|l|l|}
\hline Coding components & $\begin{array}{l}\text { The proposed } \\
\text { scheme }\end{array}$ & $\begin{array}{l}\text { The proposed + } \\
\text { displacement intra }\end{array}$ \\
\hline Sparse sample set & HEVC intra & Displacement intra \\
\hline Disparity maps & $\begin{array}{l}\text { HEVC intra } \\
\text { (lossless) }\end{array}$ & HEVC intra (lossless) \\
\hline Coding of full plenoptics & $\begin{array}{l}\text { Modified encoder } \\
\text { with HEVC intra }\end{array}$ & $\begin{array}{l}\text { Modified encoder with } \\
\text { displacement intra }\end{array}$ \\
\hline
\end{tabular}

\subsection{Decoding}

Decoding of sparse sample set: The sparse plenoptic image set is decoded.

Decoding of disparity maps: The lossless coded blockwise disparity maps and the disparity residue maps are decoded.

Disparity calculation and Reconstruction: These procedures are identical to the ones in the encoding. The two estimated sparse-set-centered disparity maps are reconstructed from the block-wise disparity maps and the residue maps. With the sparse image set and the disparity maps, a full plenoptic image is reconstructed.

Decoding of plenoptics: As an inverse process of HEVC inter frame/displacement intra encoding, a plenoptic image is decoded by using the reconstructed plenoptic image as a prediction reference.

\subsection{Scalability:}

The proposed scheme is scalable and can be viewed as having three layers. The first layer is the sparse plenoptic image set, which is in fact a sparsely sampled plenoptic image. Rendered views can be obtained directly from this image. The amount of angular information in this image depends on the sampling factor $s$. A smaller $s$ implies more angular resolution can be achieved for the rendering. The second layer is 
the reconstructed full plenoptic image given that the disparity maps are available. The reconstruction quality depends on the factor $s$ and how well the disparity estimation, the interpolation, and the inpainting are performed. The third layer is the residues of the prediction by using the reconstructed plenoptic image as a prediction reference. When these residues and their associated information are present, a required quality of the original plenoptic image in terms of PSNR can be decoded.

\section{TEST ARRANGEMENT AND EVALUATION CRITERIA}

Light field images Seagull and Fredo [15] were used in the test. These plenoptic images are densely sampled with a different depth distribution and scene. The original images have a resolution of 7240 by 5236, and the EI is of 75 by 75 with a rectangular shape. Because vignetting appears on those EIs at the corner of the plenopitc images, we cropped the center of each EI out with a size of 64 by 64 and attached them together to form the processed plenoptic images. The processed version of the image has a resolution of 6080 by 4544 . All images were transformed into $Y U V$ 4:2:0 format.

HEVC Test Model (HM) reference software version 11 was used for coding of the sparse plenoptic image set, the block-wise disparity maps and the disparity residue maps. The sparse plenoptic image set was encoded by using the HEVC original intra mode and the displacement intra with Quantization Parameters (QP) 22, 27, 32, and 37. The coding configurations are set as the "All Intra-Main" and the "Lowdelay B-Main" setting in JCTVC-L1100 [16] for the HEVC original intra and the displacement intra, respectively. The block-wise disparity maps and the residue maps were encoded by using lossless intra mode with the Coding Tree Unit (CTU) of size 16. We also modified the HEVC encoder and integrated the displacement intra into the propose scheme for the HEVC inter frame/displacement intra encoding. The current QP used for this was the same as for coding the sparse plenoptic image set, and the coding setting was the "Low-delay BMain".

The parameter for the sampling factor was $s=5$, and for the disparity estimation $r=4$. In this paper, an optimization of these parameters has not been considered. We evaluated the performance of the proposed scheme by using fixed parameters.

The quality was assessed on the luminance component with PSNR, and the bit rate, bits per pixel (bpp), was calculated from the coded bit stream for all $Y U V$ components. The rate distortion curve is plotted for PSNR vs bpp, and the BD-PSNR [17] was also computed. The proposed scheme has been compared to original HEVC intra and the displacement intra for plenoptic images.

\section{RESULTS AND ANALYSIS}

The results in Fig. 8 show that the proposed scheme reduces coding bit rate significantly than HEVC intra for all tested QPs. However, the displacement intra in HEVC still outperforms the proposed scheme for the three higher bit rate points. But, at the lowest bit rate, the proposed scheme achieves similar coding efficiency compared to the displacement intra. The results are consistent for the tested images. In addition, the BD-PSNR/rate in Table 2 illustrates that the improvement for the proposed scheme is larger than $3 \mathrm{~dB}$ compared to the original HEVC intra, or equivalently that bit rate over 40 percent is reduced.
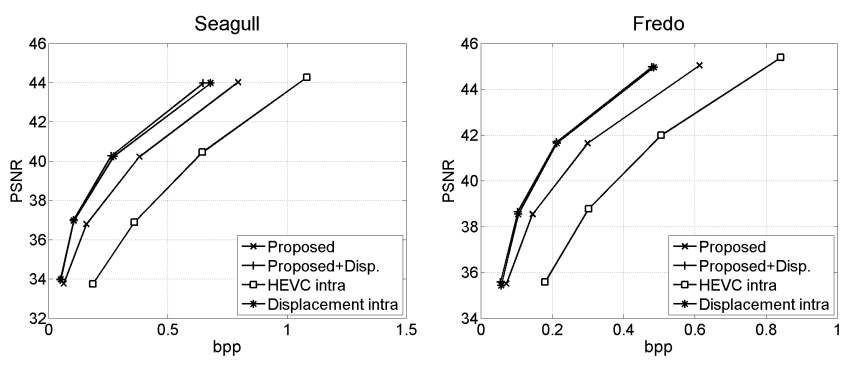

Fig. 8. Rate-distortion curves for Seagull and Fredo.

Table 2. BD-PSNR/rate: compared to HEVC intra

\begin{tabular}{|l|l|r|r|}
\hline Image & Coding methods & BD-PSNR (dB) & BD-rate (\%) \\
\hline \multirow{3}{*}{ Seagull } & Proposed & +3.19 & -48.28 \\
\cline { 2 - 4 } & Proposed+Disp. & +4.54 & -63.20 \\
\cline { 2 - 4 } & Displacement intra & +4.41 & -61.91 \\
\hline \multirow{3}{*}{ Fredo } & Proposed & +3.57 & -44.27 \\
\cline { 2 - 4 } & Proposed+Disp. & +5.06 & -58.87 \\
\cline { 2 - 4 } & Displacement intra & +4.90 & -57.85 \\
\hline
\end{tabular}

Table 3. Size (in bytes) of each coded component for the proposed scheme

\begin{tabular}{|r|r|r|r|r|}
\hline QP & $\begin{array}{l}\text { Disparity } \\
\text { maps }\end{array}$ & $\begin{array}{l}\text { Sparse sample } \\
\text { set }\end{array}$ & $\begin{array}{l}\text { Coding of full } \\
\text { Plenoptics }\end{array}$ & Total \\
\hline 22 & 17169 & 152131 & 2573297 & 2742597 \\
\hline 27 & 17169 & 90956 & 1202089 & 1310214 \\
\hline 32 & 17169 & 50964 & 476943 & 545076 \\
\hline 37 & 17169 & 26338 & 172196 & 215703 \\
\hline
\end{tabular}

Table 3 shows the coding bit rates for each coding component of the proposed scheme for Seagull. It can be noticed that the coding of full plenoptics contributes most of bits to the bit stream, while the disparity maps add least overhead to the bit stream. This suggests that an more efficient prediction in the coding of full plenoptics is essential for improving the overall coding performance. As expected, the bit rate reduction is witnessed by using the proposed+displacement intra, which surpasses the displacement intra slightly for all the tested bit rate points shown in Fig. 8. Furthermore, it can be seen in Table 2 that the bit rate reduction is above 1 percent for the 
proposed+displacement intra compared to the displacement intra.

\section{CONCLUSION}

In this paper, we have proposed a scalable coding scheme for densely sample plenoptic images. The scheme represents a full plenoptic image by its sparse sample set and associated disparity information. For the encoding, the sparse set and disparities are encoded accordingly. A full plenoptic image is then reconstructed from the sparse set and disparities by using interpolation/extrapolation and inpainting. Consequently, the reconstructed full image is utilized for a prediction to encode the original plenoptic image with a required PSNR. We further integrated a previously proposed displacement intra scheme into our proposed coding system. In addition, the proposed scheme is also scalable such that the rendering can be performed with the sparse sample set, the reconstructed plenoptic image, and the decoded plenoptic image.

The coding results demonstrated that plenoptic images were compressed efficiently by using the proposed scheme. It outperformed HEVC intra with more than $5 \mathrm{~dB}$ quality improvement or by over 60 percent bit rate reduction when measured by using BD-PSNR/rate. With the integration of the displacement intra into the proposed scheme, It reduced the bit rate by more than 1 percent compared to the displacement intra only.

\section{FUTURE WORK}

To optimize the depth estimation, the inpainting process, and the parameter space, i.e., the sampling factor $s$ and the disparity refinement range $r$ is our future research. In addition, a detail analysis of the scalability with respect to the network transmission and the rendering is also our future focus.

\section{REFERENCES}

[1] G. Lippmann, "preuves rversibles donnant la sensation du relief," J. Phys. Theor. Appl., vol. 7, no. 1, pp. 821825,1908 .

[2] Edward H. Adelson and James R. Bergen, "The plenoptic function and the elements of early vision," in Computational Models of Visual Processing. 1991, pp. 3-20, MIT Press.

[3] Todor Georgiev and Andrew Lumsdaine, "Focused plenoptic camera and rendering," Journal of Electronic Imaging, vol. 19, no. 2, pp. 021106, Apr. 2010.

[4] Xu Dong, Dai Qionghan, and Xu Wenli, "Data compression of light field using wavelet packet," in ICME '04. 2004 IEEE International Conference on, June 2004, vol. 2, pp. 1071-1074 Vol.2.
[5] M. Levoy and P. Hanrahan, "Light field rendering," in Proceedings of the 23rd annual conference on computer graphics and interactive techniques, 1996, pp. 31-42.

[6] M. Magnor and B. Girod, "Data compression for lightfield rendering," Circuits and Systems for Video Technology, IEEE Transactions on, vol. 10, no. 3, pp. 338343, Apr 2000.

[7] S. Kundu, "Light field compression using homography and 2d warping," in Acoustics, Speech and Signal Processing (ICASSP), March 2012, pp. 1349-1352.

[8] Chuo-Ling Chang, Xiaoqing Zhu, Prashant Ramanathan, and Bernd Girod, "Light field compression using disparity-compensated lifting and shape adaptation.," IEEE transactions on image processing, vol. 15, no. 4, pp. 793-806, Apr. 2006.

[9] Marcus Magnor and Bernd Girod, "Model-based coding of multi-viewpoint imagery," in Proceedings SPIE Visual Communications and Image Processing VCIP2000, 2000, pp. 14-22.

[10] Xiaoqing Zhu, Anne Aaron, and Bernd Girod, "Distributed Compression of Light Fields," Stanford University, report, online in Citeseerx.

[11] K. Nishino, Y. Sato, and K. Ikeuchi, "Eigen-texture method: Appearance compression based on 3d model," in Computer Vision and Pattern Recognition. IEEE Computer Society Conference on., 1999, vol. 1.

[12] Yun Li, Mårten Sjöström, Roger Olsson, and Ulf Jennehag, "Efficient Intra Prediction Scheme For Light Field Image Compression," IEEE International Conference on Acoustics, Speech, and Signal Processing (ICASSP), Florance, Italy, May 2014.

[13] B. Bross, W.J. Han, J.R. Ohm, G.J. Sullivan, Y.K. Wang, and T. Wiegand, "High efficiency video coding (HEVC) text specification working draft 10," JCT-VC Document, JCTVC-L1003, 2013.

[14] M. Bertalmio, A.L. Bertozzi, and G. Sapiro, "Navierstokes, fluid dynamics, and image and video inpainting," in Computer Vision and Pattern Recognition (CVPR) 2001. Proceedings of the 2001 IEEE Computer Society Conference on, 2001, vol. 1, pp. I-355-I-362 vol.1.

[15] "Todor Georgiev website," http://tgeorgiev.net/, retrieved: 08, 2013.

[16] F. Bossen, "Common HM test conditions and software reference configurations," Joint Collaborative Team on Video Coding (JCT-VC), JCTVC-L1100, 2013.

[17] Gisle Bjontegaard, "Calculation of average PSNR differences between RD-curves," ITU-T VCEG-M33, 2001. 